\title{
Erratum: Observing Light-by-Light Scattering at the Large Hadron Collider [Phys. Rev. Lett. 111, 080405 (2013)]
}

\author{
David d'Enterria and Gustavo G. da Silveira \\ (Received 25 February 2016; published 25 March 2016)
}

DOI: 10.1103/PhysRevLett.116.129901

In the original Letter, we presented a phenomenological study that demonstrated that light-by-light scattering $(\gamma \gamma \rightarrow \gamma \gamma)$ is open to study in photon-photon collisions at the Large Hadron Collider (LHC) by exploiting the large (quasi)real photon fluxes available in ultraperipheral proton-proton $(p-p)$, proton-lead $(p-\mathrm{Pb})$, and lead-lead $(\mathrm{Pb}-\mathrm{Pb})$ collisions. We used the MadGraph v.5 Monte Carlo framework to convolve the elastic $p$ and $\mathrm{Pb}$ photon fluxes with the elementary $\sigma_{\gamma \gamma \rightarrow \gamma \gamma}$ cross section, in order to generate exclusive $\gamma \gamma$ scattering events and simulate the effect of the ATLAS and CMS geometrical acceptance and typical analysis cuts. We have identified a problem in the original calculations affecting our procedure of convolution of the photon fluxes with the elementary cross section. This error resulted in computed light-by-light cross sections $\left(\sigma_{\gamma \gamma \rightarrow \gamma \gamma}^{\mathrm{excl}}\right)$ that were too low compared to our new calculation (fourth column of Table I). The corrected cross sections increase by up to a factor of 10 compared to those in the original Letter. Our new calculations have been independently cross-checked with the SuperChic v.2 code [1], finding a good agreement in the integrated and differential cross sections. Because of some changes in the resulting light-by-light kinematical distributions-plus the explicit trigger requirement of a photon with transverse momentum above $2 \mathrm{GeV}$ (mentioned in the original Letter but not actually applied in the final

TABLE I. Parameters for the $\gamma \gamma \rightarrow \gamma \gamma$ measurement in $p-p, p-\mathrm{Pb}$, and $\mathrm{Pb}-\mathrm{Pb}$ collisions at the LHC: (i) nucleon-nucleon c.m. energy $\sqrt{s_{\mathrm{NN}}}$; (ii) integrated luminosity $\mathcal{L}_{\mathrm{AB}} \cdot \Delta t$; (iii) exclusive $\gamma \gamma \rightarrow \gamma \gamma$ cross section for diphoton masses above $5 \mathrm{GeV}$; and (iv) expected number of signal counts per year after selection cuts (see text).

\begin{tabular}{lcccc}
\hline \hline System & $\sqrt{s_{\mathrm{NN}}}$ & $\begin{array}{c}\mathcal{L}_{\mathrm{AB}} \cdot \Delta t \\
(\text { per year })\end{array}$ & $\begin{array}{c}\sigma_{\gamma \gamma \rightarrow \gamma \gamma}^{\text {excl }} \\
{\left[m_{\gamma \gamma}>5 \mathrm{GeV}\right]}\end{array}$ & $\begin{array}{c}N_{\gamma \gamma}^{\text {excl }} \text { (per year) } \\
{\left[m_{\gamma \gamma}>5 \mathrm{GeV}, \text { after cuts }\right]}\end{array}$ \\
\hline$p-p$ & $1 \mathrm{TeV})$ & $1 \mathrm{fb}^{-1}$ & $105 \pm 10 \mathrm{fb}$ & 12 \\
$p-\mathrm{Pb}$ & 14 & $200 \mathrm{nb}^{-1}$ & $260 \pm 26 \mathrm{pb}$ & 6 \\
$\mathrm{~Pb}-\mathrm{Pb}$ & 8.8 & $1 \mathrm{nb}^{-1}$ & $370 \pm 70 \mathrm{nb}$ & 70 \\
\hline \hline
\end{tabular}
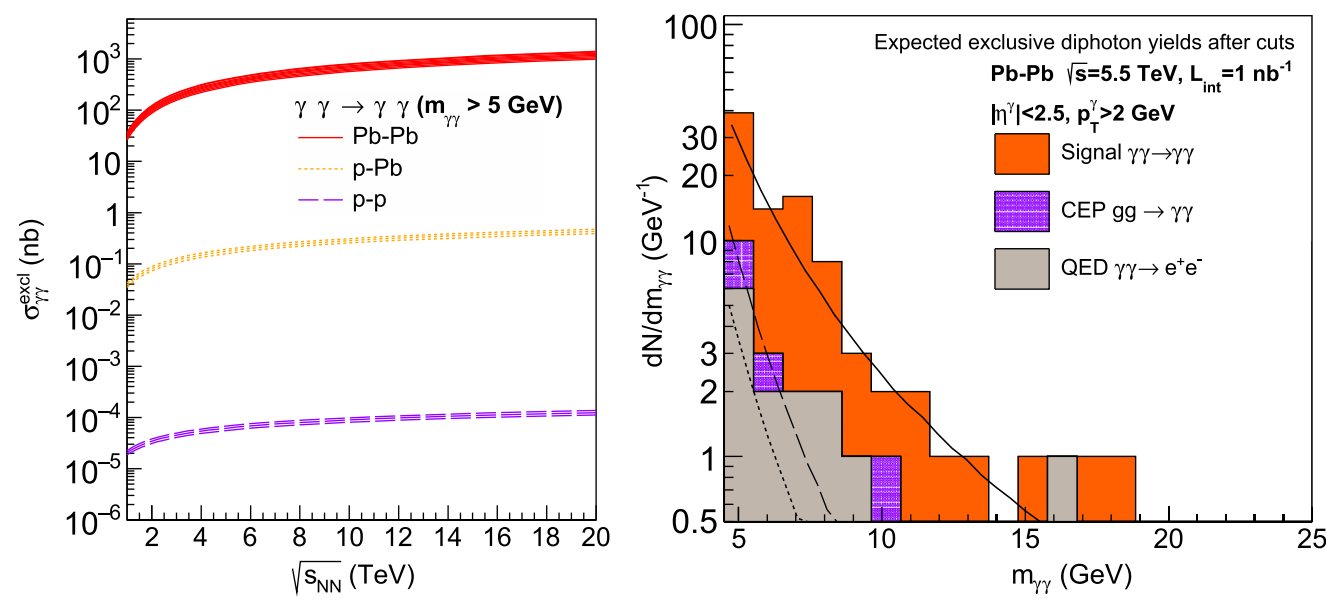

FIG. 1. Left: Cross sections for exclusive $\gamma \gamma \rightarrow \gamma \gamma$, with diphoton masses above $5 \mathrm{GeV}$, in ultraperipheral $\mathrm{Pb}-\mathrm{Pb}$ (top curve), $p$ - $\mathrm{Pb}$ (middle), and $p-p$ (bottom) collisions as a function of the nucleon-nucleon c.m. energy in the range $\sqrt{s_{\mathrm{NN}}}=1-20$ TeV. Right: Stacked diphoton yields as a function of invariant mass for exclusive $\gamma \gamma$ and backgrounds (CEP $\gamma \gamma$ and QED $e^{+} e^{-}$) expected in $1 \mathrm{nb}{ }^{-1} \mathrm{~Pb}-\mathrm{Pb}$ collisions at $\sqrt{s_{\mathrm{NN}}}=5.5 \mathrm{TeV}$ after analysis cuts (see text). The three superimposed curves indicate the individual light-by-light (solid), QED (dashed), and CEP (dotted) distributions after cuts. 
analysis there) — the corrected light-by-light yields expected after cuts $\left(N_{\gamma \gamma}^{\text {excl }}\right.$, last column of Table I) increase by about a factor of 4 compared to our original results.

In Fig. 1 (left) we plot the updated predictions for $\sigma_{\gamma \gamma \rightarrow \gamma \gamma}^{\text {excl }}$ for the three colliding systems as a function of c.m. energy $\sqrt{s_{\mathrm{NN}}}$. Figure 1 (right) shows the updated diphoton mass distribution expected for the exclusive $\gamma \gamma \rightarrow \gamma \gamma$ signal in $\mathrm{Pb}-\mathrm{Pb}$ collisions - taking into account acceptance, efficiency, and analysis cuts-normalized to the expected integrated luminosity in one $\mathrm{Pb}-\mathrm{Pb}$ run, together with the two residual central-exclusive production (CEP) and misidentified QED backgrounds. As highlighted in the original Letter, these results prove that an unambiguous observation of the process is possible in $\mathrm{Pb}-\mathrm{Pb}$ collisions which provide $\mathrm{N}_{\gamma \gamma}^{\text {excl }} \approx 70$ exclusive photon pairs per run after cuts, with controllable backgrounds. The significance of our updated result is stronger than in our original Letter, whose main conclusion, the possibility to observe light-by-light scattering in ultraperipheral lead-lead collisions at the LHC, not only does not change but is actually reinforced.

We thank Lucian Harland-Lang for valuable discussions and independent cross-checks of the $\gamma \gamma \rightarrow \gamma \gamma$ cross sections with SuperChic 2.0. G. G. S. acknowledges the support from CNPq and CAPES/Brazil.

[1] L. A. Harland-Lang, V. A. Khoze, and M. G. Ryskin, Exclusive physics at the LHC with SuperChic 2, Eur. Phys. J. C 76, 9 (2016). 